\title{
Characterization of Tablets with Defects Related to Material Sticking to Punch Faces During Pharmaceutical Manufacturing
}

\author{
A.D. Vogt, J.P. Neilly, and W. Dziki
}

Abbott Laboratories, Global Pharmaceutical Research and Development, Department R4R9, AP31, 200 Abbott Park Rd., Abbott Park, IL 60064-6202

Tablet defects include capping, lamination, chipping, cracking, binding, mottling, sticking, and picking, and there are many resources available offering corrective actions to overcome these defects. However, these are often unpredictable events that need to be dealt with as soon as possible in the development process to avoid reprocessing and costly production run delays.

Sticking is a term used to describe the adherence of tablet material to the punch faces that accumulates over time during a production run. Picking is used to describe the removal of tablet material from the surface of compressed tablets and its adherence on the punch face surface [1].

In addition to the active pharmaceutical ingredient (API), a typical formulation might include microcrystalline cellulose (binder/diluent), colloidal silicon dioxide (glidant), croscarmellose (disintegrant), and magnesium stearate (lubricant). A lubricant's main purpose is to minimize a compressed tablet's propensity to adhere to components of the tabletting machine, including the punches and dies. Adjusting the amount of lubricant can help mitigate sticking, but adding too much lubricant can affect disintegration and dissolution (i.e., bioavailability).

Tablets with different levels of lubricant and density were studied. Sticking of tablet material to the punches and dies during tabletting led to tablets with depressions that got worse the longer the tabletting run progressed (Figure 1). Characterization of the punch face residues suggest that most of the adherent material is API. Tablets with depressions from similar punch faces were characterized by scanning electron microscopy (SEM), x-ray mapping by energy dispersive x-ray spectroscopy, x-ray photoelectron spectroscopy (XPS), and atomic force microscopy (AFM).

Tablets exhibiting the greatest depression had high solid fraction (HSF) and $1.5 \%$ magnesium stearate. Those exhibiting a moderate depression had medium solid fraction (MSF) and $2.0 \%$ magnesium stearate, and those exhibiting no depression had MSF with $2.0 \%$ magnesium stearate. $\mathrm{X}$-ray mapping of depressed and non-depressed areas of these tablets indicated uniform distribution of API, magnesium stearate, silicon dioxide (aerosil), and croscarmellose across the areas mapped. Magnesium maps indicated the presence of discrete magnesium stearate particles, which might suggest under blending. XPS of the depressions in the tablets was performed to assess the relative level of components in this region compared to a tablet without these depressions (Figure 2). XPS results suggest the API was more concentrated in the tablet with the greatest depression, but no differences in API distribution were observed in the tablets with smaller or no depressions. Magnesium stearate levels were lower and aerosil levels were higher in the depressions than on a tablet without depressions.

Finally, adhesion forces between an AFM tip and the picked tablets were measured. Initial results indicated measured differences with high variability in adhesion forces between the AFM tip and 
tablets (Figure 3). These variances are in part due to tablet and particle surface roughness and tablet dust adhering to AFM tip and cantilever. Once these roughness and debris issues are addressed, AFM can be a powerful technique to understand adhesion forces between the formulation components and tooling in its relationship to sticking and picking.

The studies indicated that X-ray mapping and XPS are powerful techniques to assess tablets that were picked by punch faces. While X-ray mapping and XPS identified components and their distributions in the tablets, AFM can be used to assess formulation components tendency to adhere to tooling.

The impact of defects such as sticking and picking on manufacturing underscore the importance of having a fundamental understanding of material properties associated with tablet components and their interactions with manufacturing equipment. Prior knowledge and appropriate use of such properties can play a significant role in formulating and manufacturing pharmaceutical tablets that mitigate sticking, picking, and other common defects.

\section{Reference}

[1] M. D. Tousey, Tablets and Capsules, October 2003
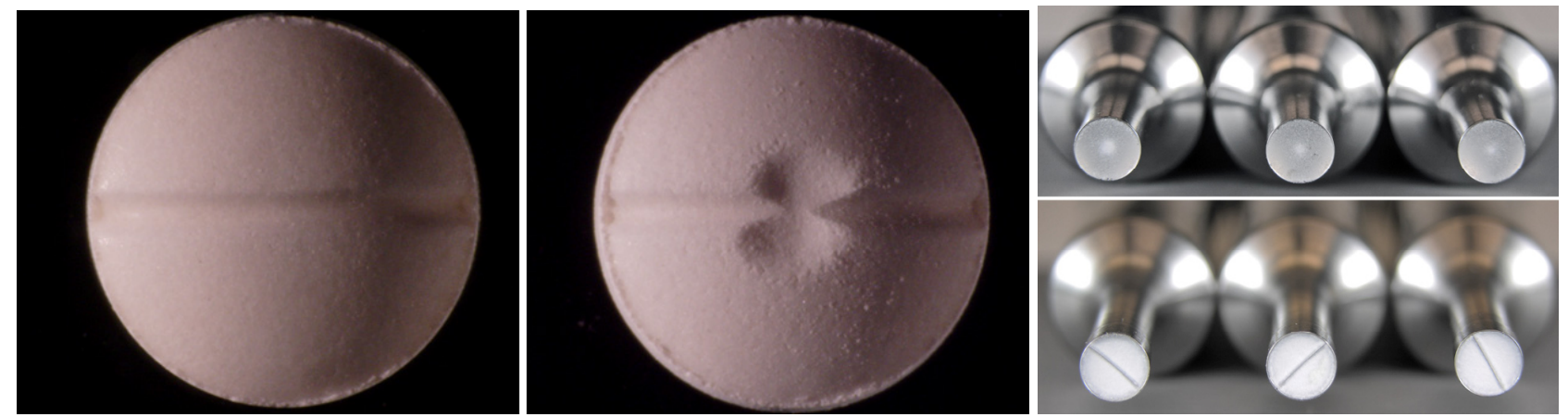

Figure 1. Left panel: normal tablet; Middle panel: tablet with depression; Right panel: Upper punch faces (top) and lower (bottom) punch faces after 500 cycles that have adhered tablet material.

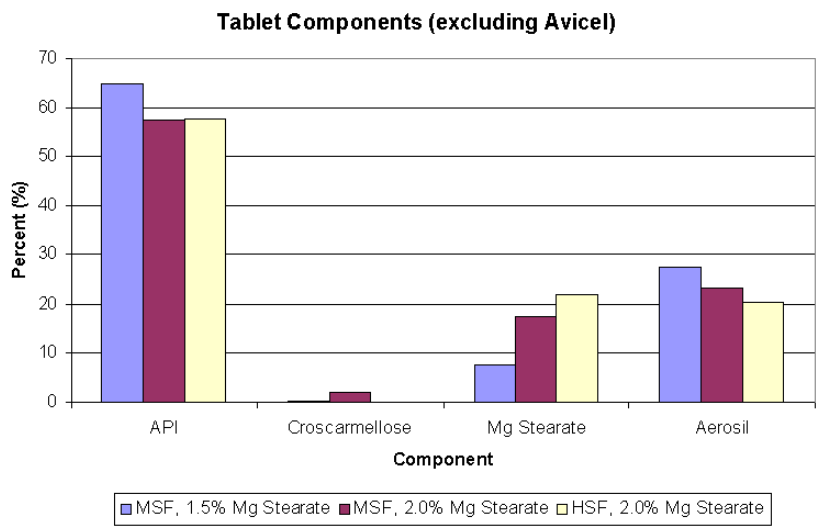

Figure 2. XPS results indicate less lubricant and more aerosil in the depressions caused by picking.

\begin{tabular}{lccc}
\hline & $\begin{array}{c}\text { HSF } \\
\text { 2\% MgSt }\end{array}$ & $\begin{array}{c}\text { MSF } \\
\text { 2\% MgSt }\end{array}$ & $\begin{array}{c}\text { MSF } \\
\mathbf{1 . 5 \%} \mathbf{~ M g S t}\end{array}$ \\
\hline Average & 10.322 & 11.398 & 9.418 \\
St. Dev. & 2.553 & 4.635 & 2.669 \\
Median & 9.682 & 13.010 & 9.453 \\
Number & 13 & 12 & 12 \\
\hline
\end{tabular}

HSF/MSF $=$ high or medium solid fraction

Figure 3. Adhesion forces in $\mathrm{nN}$ between AFM tip and tablets. 